
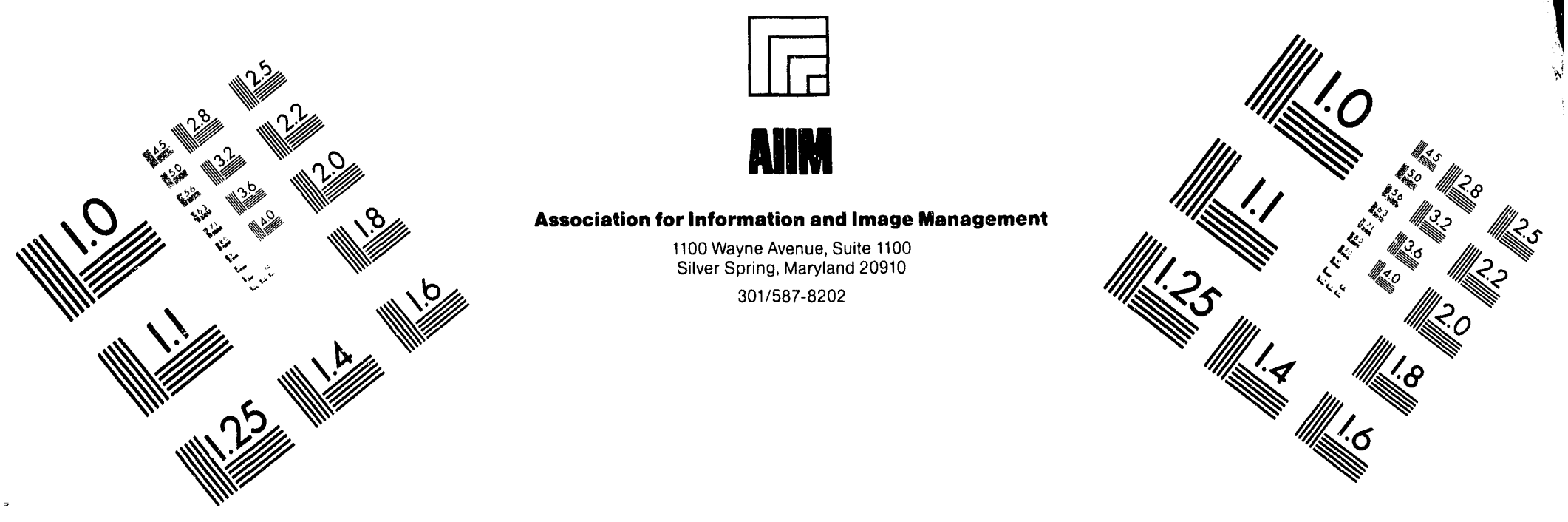

\title{
Centimeter
}

| Inches
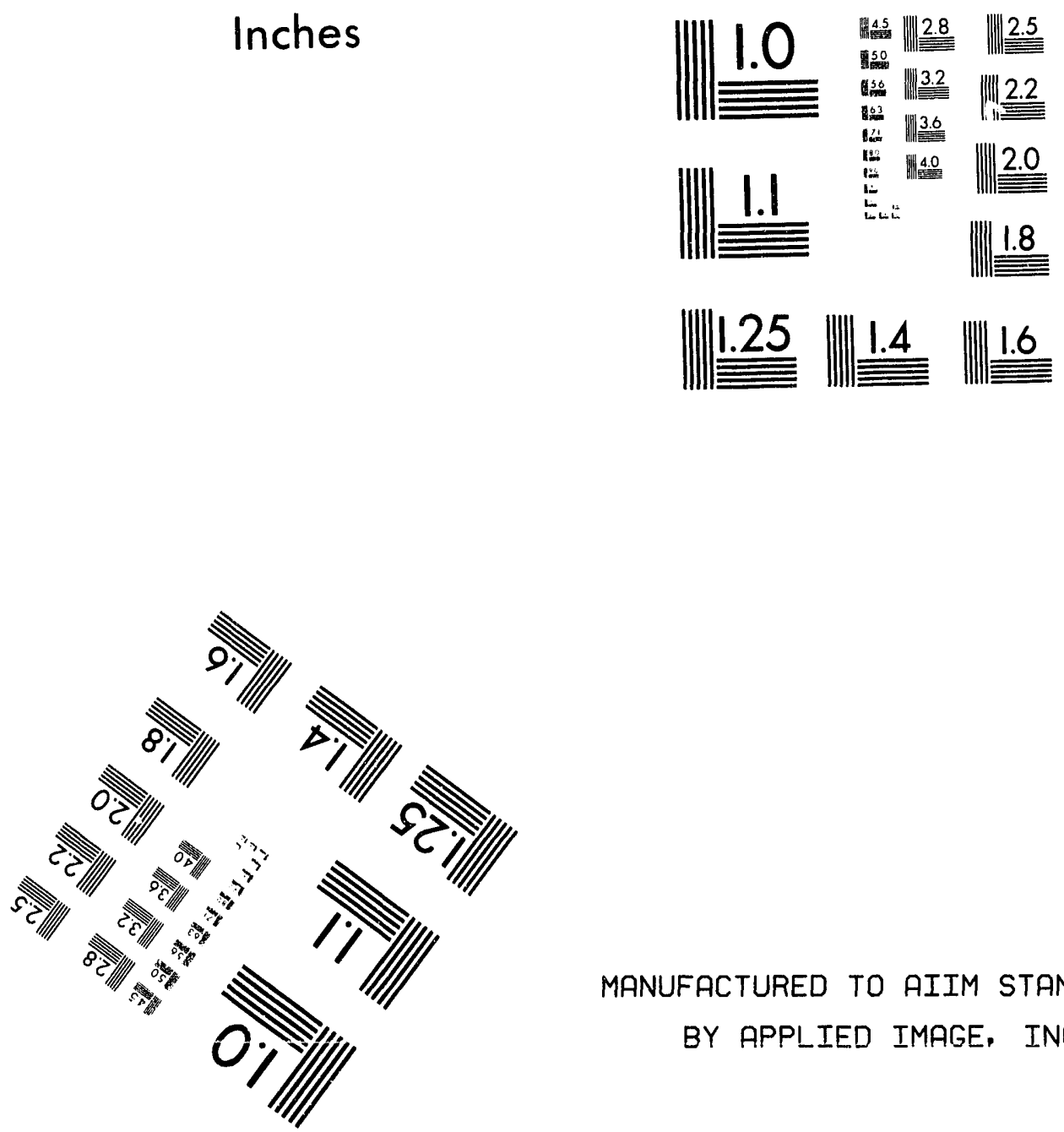

MANUFACTURED TO AIIM STANDARDS

BY APPLIED IMAGE, INC.

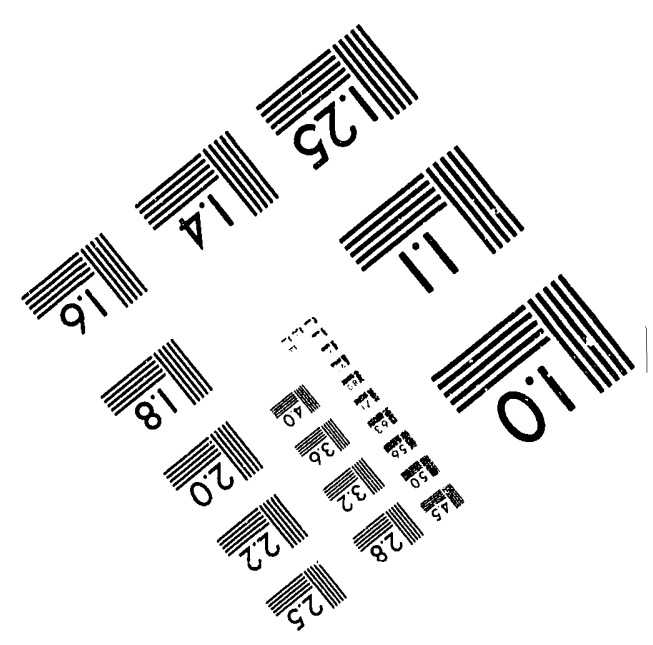



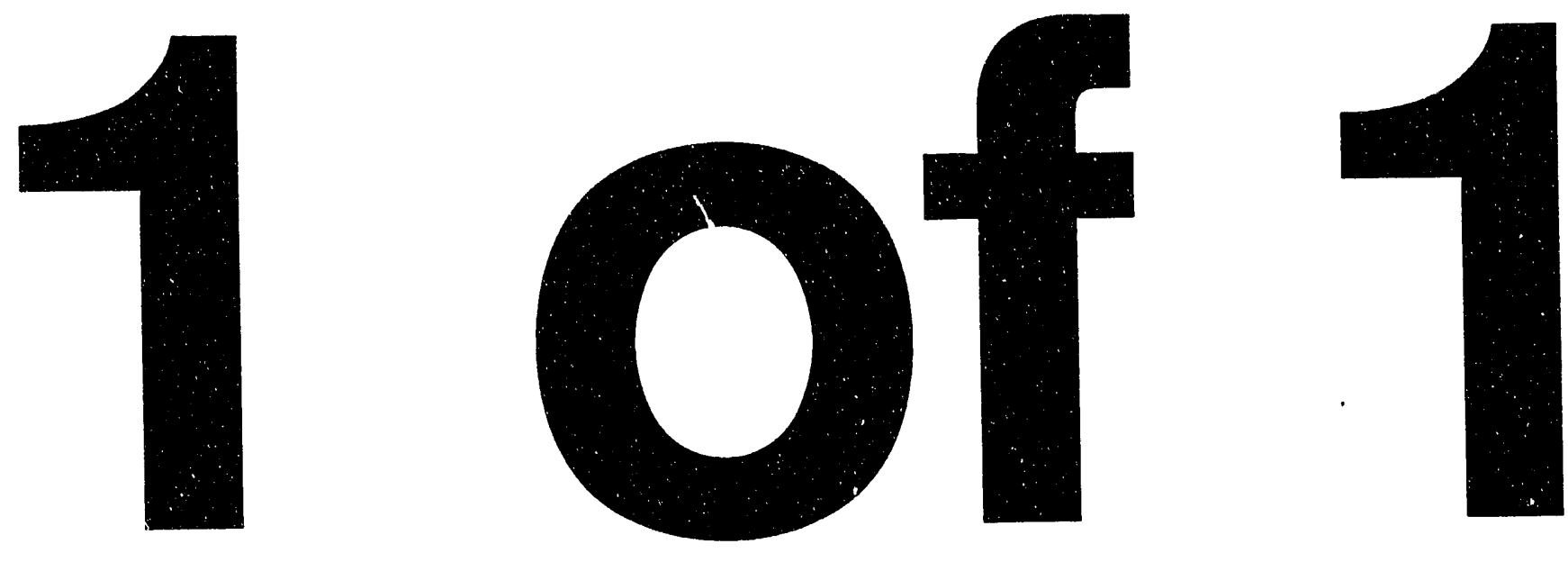


\section{Required Cavity HOM deQing Calculated from Probability Estimates of Coupled Bunch Instabilities in the APS Ring*}

\section{H. JUN 141993 OSTI}

A method of determining the deQing requirement of individual cavity higher-order modes (HOM) for a multi-cavity RF system is presented and applied to the APS ring. Since HOM resonator frequency values are to some degree uncertain, the HOM frequencies should be regarded as random variables in predicting the stability of the coupled bunch beam modes. A Monte Carlo simulation provides a histogram of the growth rates from which one obtains an estimate of the probability of instability. The damping of each HOM type is determined such that the damping effort is economized, i.e. no single HOM dominates the specified growth rate histogram.

\section{INTRODUCTION}

Previous work [1] on coupled-bunch instability in the APS ring consisted of calculating the growth rate of coupled bunch modes (CBM) assuming that only one HOM from one cavity was shifted to the frequency of the closest CBM. The probability that HOMs from two cavities contributed to the same CBM was deemed small because the HOM frequencies from different cavities were staggered. The required deQing for each monopole and dipole HOM type was derived from these results.

A possibility that should now be considered in a staggered HOM frequency RF system is that once the HOMs are deQed, the impedance functions of two HOMs close in frequency might overlap, thus negating frequency staggering. Another possibility is two or more HOM impedances from different HOM types contributing to the same CBM through frequency aliasing.

In order to cover all possibilities, one has to calculate the CBMs using all of the HOM impedances with staggered frequencies. Since the HOM resonant frequency values have a quasi-random nature, a statistical analysis of the CBM growth rates is used to estimate the amount of required HOM deQing for beam stability. A similar Monte Carlo analysis was first reported by Sicmann in [2].

The relevant data in the instability calculation are given in Section II. Section III explains the Monte Carlo method used, and gives a prescription for determining minimum HOM deQing factors. The method is applied to the APS ring in Section IV. As one may expect, the resulting deQing requirement is more stringent than the preliminary one reported in [1].

All coupled bunch growth rates are calculated by the new program clinchor [3]. It uses a normal mode analysis based on the theory of Thompson and Ruth [4]. One of clinchor's

\footnotetext{
* Work supported by U.S. Department of Energy, Office of Basic Energy Sciences under Contract No. W-31-109-ENG-38.
}

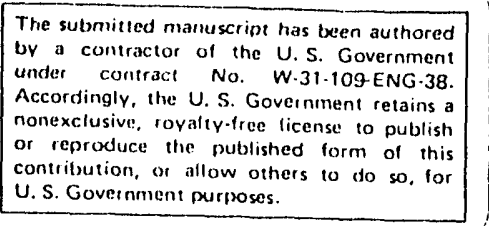

features is the possibility of randomizing the HOM frequencies, an idea borrowed from the program PC-BBI [5].

\section{RING PARAMETER AND CAVITY HOM DATA}

Some of the ring parameters used in the calculations are listed in Table 1. These parameters are constant for all calculations presented here. The $\beta$ functions at the RF cavities are used instead of the transverse tunes for the transverse growth rate calculation.

Table 1

General parameters for growth rate calculations.

\begin{tabular}{|ll|cl|}
\hline Ring energy & $E$ & 7 & $\mathrm{GeV}$ \\
\hline Total current & $I_{\mathrm{t}}$ & 300 & $\mathrm{~mA}$ \\
\hline Number of bunches & $N$ & 54 & \\
\hline Current per bunch & $I_{\mathrm{b}}$ & 5.6 & $\mathrm{~mA}$ \\
\hline Number of cavities & $N_{\mathrm{c}}$ & 16 & \\
\hline Revolution frequency & $f_{0}$ & 271.55 & $\mathrm{kHz}$ \\
\hline Synchrotron frequency & $f_{\mathrm{s}}$ & 1.5 & $\mathrm{kHz}$ \\
\hline Longitudinal damping rate & $1 / \tau_{\varepsilon}$ & 213 & $\mathrm{sec}^{-1}$ \\
\hline Transverse damping rate & $1 / \tau_{x}$ & 106 & $\mathrm{sec}^{-1}$ \\
\hline$\beta_{x}$ at RF cavities & & 14 & $\mathrm{~m}$ \\
\hline$\beta_{y}$ at RF cavities & 10 & $\mathrm{~m}$ \\
\hline
\end{tabular}

The properties of the APS ring RF cavity HOMs, as calculated by the program URMEL [6], are listed in Table 2. Each HOM type is named after its unperturbed resonant frequency listed in the first column. $R_{S}$ is the longitudinal shunt impedance, and $R_{l}$ is the transverse shunt impedance. Later, $R$ will simply refer to the shunt impedance in general. $Q_{0}$ is the unperturbed quality factor of the HOM resonator. To explain the last column, staggering the HOM frequencies means separating the frequencies by equal intervals. This is accomplished by designing each cavity longer than the previous one by some small amount. The effect is different for each HOM type because of their distinct field patterns. The staggering interval column in Table 2 lists how much the HOM resonant frequency decreases per cavity using $0.3 \mathrm{~mm}$ per cavity as the clongation step. The selection of the elongation step, according to [7], is based on the budgeted tuning range $(0.75 \mathrm{MHz})$ of the cavity tuners to correct for the fundamental mode frequency staggering. 
Table 2

Impedance parameters of HOMs.

\begin{tabular}{|c|c|c|c|}
\hline $\begin{array}{c}\text { Monopole HOMs } \\
(\mathrm{MHz})\end{array}$ & $\begin{array}{c}Q_{0} \\
(1000)\end{array}$ & $\begin{array}{c}R_{s} \\
(\mathrm{M} \Omega)\end{array}$ & $\begin{array}{c}\text { Staggering } \\
\text { interval }(\mathrm{kHz})\end{array}$ \\
\hline $352^{*}$ & 49 & 5.60 & 5.1 \\
\hline 538 & 41 & 1.67 & 210. \\
\hline 922 & 107 & 0.62 & 330. \\
\hline 939 & 42 & 0.23 & 330. \\
\hline 1172 & 44 & 0.18 & 390. \\
\hline 1210 & 94 & 0.49 & 600. \\
\hline 1509 & 88 & 0.40 & 810. \\
\hline
\end{tabular}

\begin{tabular}{|c|c|c|c|}
\hline $\begin{array}{c}\text { Dipole HOMs } \\
(\mathrm{MHz})\end{array}$ & $\begin{array}{c}Q_{0} \\
(1000)\end{array}$ & $\begin{array}{c}R_{\mathbf{t}} \\
(\mathrm{M} \Omega / \mathrm{m})\end{array}$ & $\begin{array}{c}\text { Staggering } \\
\text { interval }(\mathrm{kHz})\end{array}$ \\
\hline 588.7 & 68 & 13.6 & 24. \\
\hline 761 & 53 & 25.6 & 210. \\
\hline 962 & 54 & 6.1 & 360. \\
\hline 1017 & 41 & 2.6 & 510. \\
\hline 1145 & 92 & 2.7 & 450. \\
\hline 1219 & 41 & 3.6 & 570. \\
\hline
\end{tabular}

* Fundamental mode included for comparison.

Due to its longitudinal symmetry, the staggering of the $588-\mathrm{MHz}$ dipole HOM is relatively small. The 16 impedance functions of this HOM type will overlap, requiring this HOM type to be damped more strongly than others.

In heavily beam-loaded RF systems, there is a concem that the fundamental mode couple to the longitudinal CBMs of frequencies $f_{0}+f_{s}$ and $(N-1) f_{0}+f_{s}$. In our case, the coupling is negligible. Not included in the transverse CBM growth rates is the effect of the resistive wall impedance. Since this impedance component is not RF related, it is ignored for now.

\section{STATISTICAL ANALYSIS OF STABILITY}

A multi-bunch beam is predicted to be unstable in a storage ring if the growth ratc of any CBM calculated for a given impedance is greater than the natural damping rate (here the radiation damping rate) of the beam. Since the damping rate is fixed for each plane of motion, one only necds to calculate the largest growth rate among the CBMs to determine stability of the beam in each plane.

The growth rate is a highly sensitive function of the HOM frequencies. However, the HOM frequencies are uncertain quantities, since they change during normal operation of a ring. In addition, before the assembly of cavities, one does not know by how much the construction errors will shift the HOM frequencies from their expected staggered values. Thus, the growth rate is an uncertain quantity.

Since beam stability is directly related to growth rate values, one therefore deals with probability of beam stability. One defines the probability of beam stability to be the number of sets of possible HOM frequencies where the beam is stable divided by the total number of scts of possible HOM frequencies.

Obviously, one must approximate the probability calculation by restricting the infinite set of HOM frequencies to a manageable few. The analysis thus becomes a statistical one.

The Monte Carlo simulation proceeds as follows. One establishes a set of fixed HOM frequencies to which are then added random values in a range given by an error model. A value for the largest growth rate among the CBMs is stored for each set of HOM frequencies generated.

The set of growth rate values can be histogrammed to show their distribution (sec Figure 1, for example). The shape of the histogram is a function of the HOM $R$ 's (here, $R$ just means shunt impedance for cither plane of motion), $Q$ 's, and $f$ 's and of the random values amplitude. Typically, a sample of 50 random sets of randomized HOM frequencies is sufficient to provide a smooth enough histogram.

A consistent criterion for reasonable certainty of beam stability is now given: if the 95th percentile value of growth rates is greater than the natural damping rate, then the beam is stable (i.e. the beam is stable $95 \%$ of the time); otherwise the beam is unstable.

The HOM deQing requirements are obtained as follows, recognizing that each HOM type has differing $R / Q$ values, and one would like to economize the minimum damping effort. As a first step, each HOM type is taken as being the only impedance in the ring. If the beam is predicted by the Monte Carlo calculation to be unstable, the input values of $R$ and $Q$ of the HOMs are reduced such that a repeated Monte Carlo calculation predicts a stable beam. During this step, one observes that growth rates are not necessarily proportional to $R$ since, by reducing $Q$, the impedance functions spread and couple to more CBMs. Thus a few iterations may be required before determining the deQing factor for cach HOM.

In the last step, all HOMs are included together with their new $Q$ 's, and all are further deQed with the same factor for beam stability.

\section{APPLICATION TO APS RING}

A simple frequency error model is adopted here because the correct random component of the HOM frequencies attributable to construction errors and operating circumstances is difficult to cstablish.

A conservative range of $\pm f_{0}$ ( $f_{0}$ is the separation of dangerous frequencies) is selected for the random component of all HOMS. That is, it is guarantecd that some frequency values will land very close to a resonance. This prevents the frequency samples from repeating "safe" values of frequency in between resonances. If the actual random component is larger than $f_{0}$, the CBMs that are influenced by the impedances will be different ones. However, the distribution of growth rates is not expected to change much.

Table 3 lists the deQing factors at the first stage of the analysis for dipole HOMs. (Because of space limitations, the table for monopole HOMs is not shown). 
Table 3

DeQ factor for cach HOM type taken separately.

\begin{tabular}{|c|c|c|c|}
\hline $\begin{array}{c}\text { Dipole } \\
\text { HOMs } \\
(\mathrm{MHz})\end{array}$ & $\begin{array}{c}\text { Damping } \\
\text { factor } \\
Q^{\prime} Q_{0}\end{array}$ & $\begin{array}{c}\text { Resonator } \\
\text { width } \\
(\mathrm{MHz})\end{array}$ & $\begin{array}{c}\text { Staggering } \\
\text { range } \\
(\mathrm{MHz})\end{array}$ \\
\hline 588 & 150 & 1.300 & 1.2 \\
\hline 761 & 220 & 3.100 & 10.5 \\
\hline 962 & 9 & 0.161 & 18. \\
\hline 1017 & 2.5 & 0.045 & 26. \\
\hline 1145 & 2.5 & 0.031 & 23. \\
\hline 1219 & 3 & 0.089 & 29. \\
\hline
\end{tabular}

The resonator width is $f_{\mathrm{HOM}} / Q$. The staggering range is the spread of HOM frequencies of the cavities. For the 16 cavities, it is just 15 times the staggering interval in Table 2. For the two lower frequency HOMs, the resonance width is of the order of the staggering range, implying that some of the impedance functions from the 16 cavities overlap, and that damping effort becomes greater. For the other HOMs in the table, there is no obvious overlapping of impedances.

When all HOM types are combined, the probability of two HOM frequencies for contributing to the same CBM is increased through aliasing of the resonant frequencies.

Table 4

Required $Q$ for frequency-staggered cavity HOMs.

\begin{tabular}{|c|c|c|}
\hline $\begin{array}{c}\text { Monopole HOM type } \\
\text { (MHz) }\end{array}$ & $\begin{array}{c}\text { DeQing factor } \\
Q^{\prime} Q_{0}\end{array}$ & $\begin{array}{c}\text { Required } \\
Q\end{array}$ \\
\hline 538 & 64 & 640 \\
\hline 922 & 16 & 6700 \\
\hline 939 & 6.4 & 6600 \\
\hline 1173 & 6.4 & 6900 \\
\hline 1210 & 16 & 5900 \\
\hline 1509 & 16 & 5500 \\
\hline
\end{tabular}

\begin{tabular}{|c|c|c|}
\hline $\begin{array}{c}\text { Dipole HOM type } \\
(\mathrm{MHz})\end{array}$ & $\begin{array}{c}\text { DeQing factor } \\
Q^{\prime} Q_{0}\end{array}$ & $\begin{array}{c}\text { Required } \\
Q\end{array}$ \\
\hline 588 & 285 & 240 \\
\hline 761 & 420 & 130 \\
\hline 962 & 17 & 3200 \\
\hline 1017 & 4.8 & 8500 \\
\hline 1145 & 4.8 & 19200 \\
\hline 1219 & 5.7 & 7200 \\
\hline
\end{tabular}

The final values of $Q / Q_{0}$ are listed in Table 4. The required $Q$ column is the maximum $Q$ allowed for stability. Figure 1 shows the histogram and its integral of the growth rates for the final set of $R_{s}$ 's and $Q$ 's for the longitudinal case. The dashed line is the synchrotron radiation damping rate. Note that, for most samples, the growth rate due to the HOM impedances is only about half the damping rate.
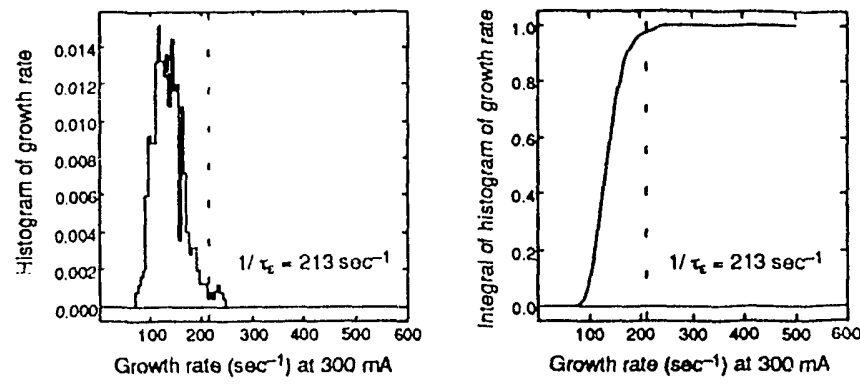

Figure 1

Final histogram and its integral for the longitudinal case.

\section{CONCLUSION}

The HOM deQing requirements for frequency-staggered cavities have been calculated based on the probability of beam stability using HOM resonator frequencies as random variables.

The deQing requirement for some of the dipole HOMs is stringent because their frequency staggering is small and their $R_{f} / Q$ is particularly high.

\section{REFERENCES}

[1] L. Emery, "Coupled Bunch Instabilities in the APS Ring," Proceedings of the 1991 IEEE Particle Accelerator Conference, 1991 .

[2] R. Sicmann, "Instability Growth Rate Calculations for High Energy Storage Rings," Proceedings of the 1981 IEEE Particle Accelerator Conference, Vol. NS-28, p. 2437, June 1981.

[3] L. Emery, "User's Guide to Program clinchor," ANL technical report, to be published.

[4] K. Thompson and R. Ruth, "Transverse and Longitudinal Coupled Bunch Instabilities in Trains of Closely Spaced Bunches," Proceedings of the 1989 IEEE Particle Accelerator Conference, p. 792, 1989.

[5] J. Hagel and B. Zotter, "PC-BBI, a Program to Compute Bunch Bcam Instabilitics on a PC," CERN SL-AP 90-62, CERN, 1990.

[6] T. Wciland, "On the Computation of Resonant Modes in Cylindrically Symmetric Cavities," NIM, Vol. 216, pp. 329-348, 1983.

[7] Y. Kang, private communication.

\section{DISCLAIMER}

This report was prepared as an account of work sponsored by an agency of the United States Government. Neither the United States Government nor any agency thereof, nor any of their employees, makes any warranty, express or implied, or assumes any legal liability or responsibility for the accuracy, completeness, or usefulness of any information, apparatus, product, or process disclosed, or represents that its use would not infringe privately owned rights. Reference herein to any specific commercial product, process, or service by trade name, trademark, manufacturer, or otherwise does not necessarily constitute or imply its endorsement, recommendation, or favoring by the United States Government or any agency thereof. The views and opinions of authors expressed herein do not necessarily state or reflect those of the 

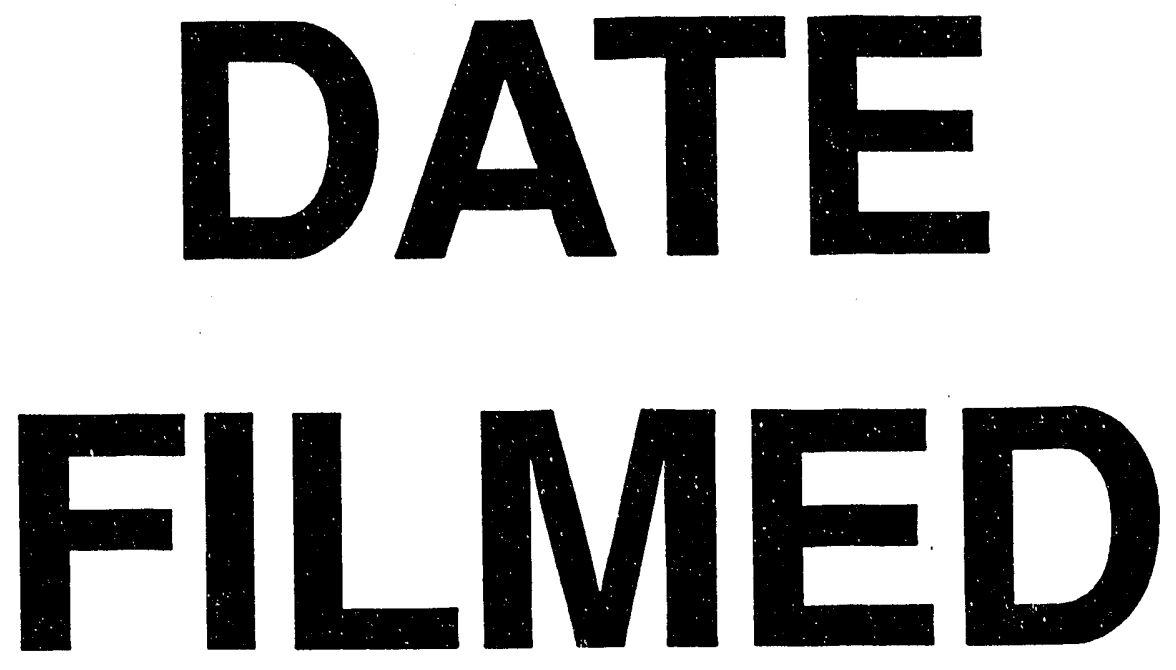

$8 / 16 / 93$
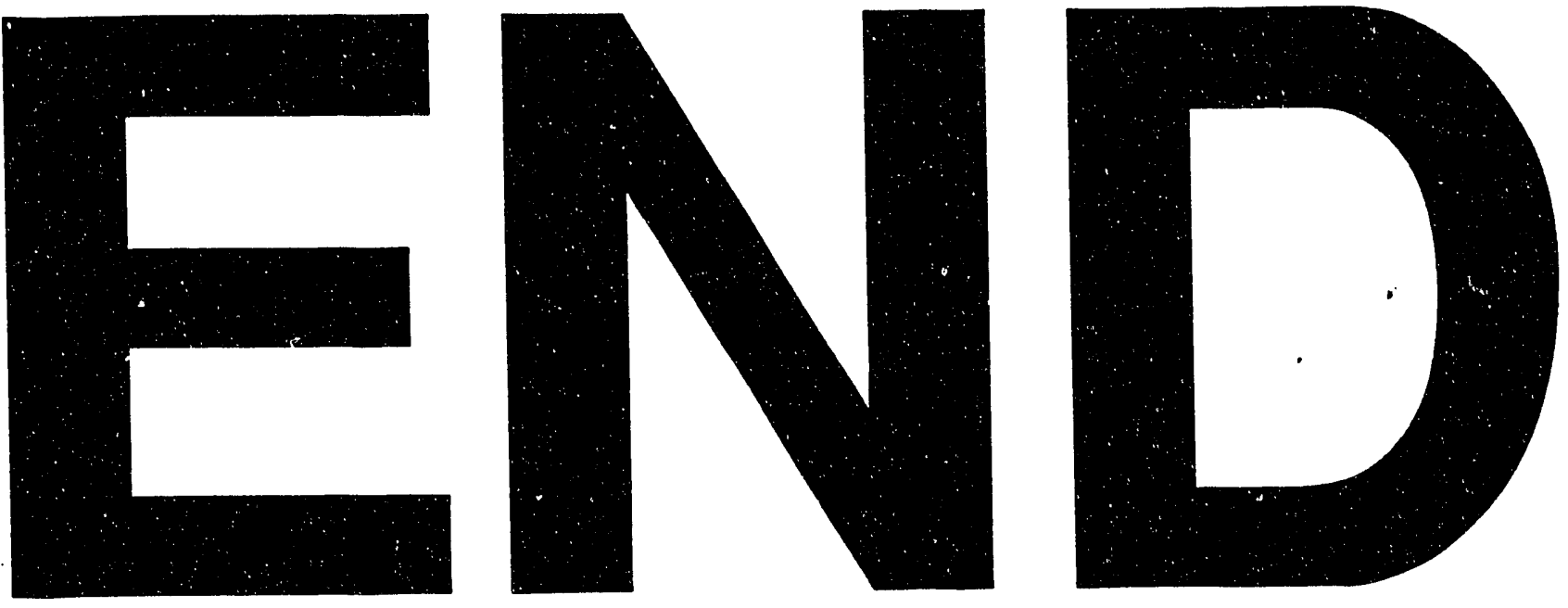
